\title{
Evaluation of Nonlinear Interference Effects in a Dispersion Managed(DM) Optical Fiber: Performance and Transmission Analysis of 16QAM Modulation using Split Step Fourier Method
}

\author{
Reinhardt Rading \\ Department of Engineering and Architecture \\ University of Parma \\ Parma, Italy
}

\begin{abstract}
This paper investigates the impact on the optical signal-to-noise ratio (OSNR) of the residual per span (RDPS) in a $\mathbf{N} \times 100 \mathrm{~km}$ dispersion managed system with zero total accumulated dispersion from input to output using split step Fourier method (SSFM) -Monte Carlo simulation. This paper will show that the nonlinear interference NLI does in-fact impact the performance yielding different best working power depending on the value of $N \times 100 \mathrm{~km}$ span and the type of dispersion managed link. The paper will show that dispersion uncompensated optical links are preferable to dispersion managed fibers in equalizing NLI effects in long haul optical links.
\end{abstract}

Index Terms-Optical Signal-to-Noise Ratio(OSNR), Dispersion Managed(DM), Residual per Span(RDPS), Nonlinear Interference (NLI)

\section{INTRODUCTION}

Due to huge data demand and the need for efficient spectrum usage, coherent systems are becoming the norm for long haul optical transmission systems and network. After several of kilometers of transmission, the optical system experiences signal degradation resulting from the noise produced in the system. One metric used to characterize degradation and measure the quality of transmission (QoT) is the use of optical signal-to-noise Ratio (OSNR)- defined in optical domain as the ratio of optical signal power to noise power.

Different models [1] have been suggested including for example,the Gaussian Noise (GN) model [2], used to predict performance of an optical link in the nonlinear regime. Apart from the amplified spontaneous (ASE) noise, nonlinear signal interference (NLI)-a noise like power induced by nonlinear effects -is factor that contributes to signal degradation in long haul optical system. Many models assumes that NLI is a small perturbation of nonlinear Schrodinger equation [3] but it has an immense effect on nonlinear propagation and thus should be included in QoT prediction.

The aim of this paper is to show that NLI does in-fact impact the performance,yielding different working power depending on distance and the dispersion on the link. The paper is divided into five sections: section II discusses the theoretical part, section III explains the numerical analysis, section IV states the simulation results, and section $\mathrm{V}$ discusses the simulation results. The paper finally draws conclusion in section VI.

\section{THEORY}

An optical transmission system traversing several of kilometers will experience signal impairments resulting from ASE noise (results from signal amplification using Erbium doped fiber amplifiers) and nonlinear noise (results from nonlinear fiber response to the input power). Signal distortion due to nonlinear fiber response increases with an increase in input power, increase in transmission length, and transmission rate.

High power in optical systems results to a change in the material refractive index with respect to the electric field intensity leading to a phenomenon known as Kerr Effect [4]. The Kerr Effects leads to signal distortion and limits the distance [5] the signal can travel without experiencing distortion.

For an $\mathrm{N}$ span system, the total NLI is the sum of $\mathrm{N}$ noise random variables. Gaussian model assumes that the noise contributions are uncorrelated and thus ease of use and to determine their influence. The limitation of Gaussian model is that the above assumption only holds over in a dispersion uncompensated optical links.

This paper focuses on dispersion managed links with dispersion compensating fibers (DCF) being used to compensate for dispersion in the link and are applied pre-transmission, inline or post transmission. Different dispersion managed (DM) links exists with a 0 residual dispersion per span (0 RDPS) or a 30 residual dispersion per span (30 RDPS).

Non-linear effects in a fiber can be determined by solving the Non-Linear Schrondiger Equation given by equation 1 using split step Fourier method (SSFM). Compared to other NLSE models, SSFM is two magnitude orders faster [6].

$$
\frac{d A}{d Z}=-\frac{\alpha}{2} A-B_{1} \frac{d A}{d t}+j \frac{\beta_{2}}{2} \frac{d^{2} A}{d t^{2}}+\frac{\beta_{3}}{6} \frac{d^{3} A}{d t^{3}}-j \gamma|A|^{2} A
$$


Where $\mathrm{A}$ is the slowly varying amplitude of the field, $\alpha$ is the loss coefficient of the fiber, $\beta_{1}, \beta_{2}$, and $\beta_{3}$ are the first, second and third order dispersion coefficients of the fiber, respectively. $\gamma$ is the nonlinear coefficient of the fiber (an equivalent value of $1.37 / \mathrm{W} / \mathrm{Km}$ in SMF fibers of $80 \mathrm{~mm}^{2}$ ).

NLI includes self-phase modulation (SPM), cross phase modulation (XPM), and four wave mixing (FWM). For a wavelength division multiplexed systems, the nonlinear Schrondiger equation [3] is shown in equation 2.

$$
\begin{aligned}
\frac{d A_{n}}{d Z}=-j B_{1 n} \frac{d A_{n}}{d Z} & +j \frac{\beta_{2 n}}{2} \frac{d^{2} A_{n}}{d t^{2}}+\frac{\beta_{3 n}}{6} \frac{d^{3} A_{n}}{d t^{3}} \\
& -j \gamma \Sigma A_{k} A_{l} A_{m} e^{-j \Delta B_{k l m} Z}
\end{aligned}
$$

where

$$
\Delta B_{k l m n}=\beta_{w k}+\beta_{w l}-\beta_{w n}-\beta_{w m}
$$

If $k=l=m=n$, then $\Delta B_{k l m n}=0$ and the optical link experiences self phase modulation. If $(k=l) \neq(m=n)$, then $\Delta B_{k l m n}=0$, the system experiences cross phase modulation. Otherwise, $\Delta B_{k l m n} \neq 0$ and the optical link experiences four wave mixing [7].

\section{NUMERICAL ANALYSIS}

The fiber parameters used for simulation included a SMF $($ Dispersion $=17 \mathrm{ps} / \mathrm{nm} / \mathrm{km}$, nonlinear coefficient $=1.3 / \mathrm{W} / \mathrm{Km})$ optical link with a single channel with an input power of $1 \mathrm{dBm}$, loss coefficient of $0.2 \mathrm{~dB} / \mathrm{Km}, \mathrm{N}$ spans with each span being $100 \mathrm{~km}$, an amplifier of a $6 \mathrm{~dB}$ noise figure, a symbol rate of $10 \mathrm{~Gb} / \mathrm{s}$ under a 16QAM modulation format. The DCF had a loss coefficient of $0.6 \mathrm{~dB} / \mathrm{Km}$. The fiber propagation was modelled using Manakov equation and the simulation ran on Optilux [8] under Split Step Fourier-Monte Carlo simulation model.

The first simulation was done on an input power of $1 \mathrm{dBm}$ with an RDPS of $0 \mathrm{ps} / \mathrm{nm}$. The simulation was repeated with a variation of power between $-22 \mathrm{dBm}$ to $10 \mathrm{dBm}$ while holding other parameters the same as indicated above. The value of RDPS was changed to 30 and $70 \mathrm{ps} / \mathrm{nm}$ while keeping the value of other parameters as previously. The power was varied to the specified range for each RDPS value. The second simulation involved using a WDM system with 5 channels with a spacing of $37.5 \mathrm{GHz}$ with similar procedures as above.

\section{Simulation Results}

For a single channel optical link with 0 RDPS, a reduction in OSNR was observed with an increase in the number of spans. For example, with an input power of $1 \mathrm{dBm}$, the OSNR after one span was $30.03 \mathrm{~dB}$ whereas after ten spans, the OSNR recorded was $17.25 \mathrm{~dB}$. If the power was increased to $10 \mathrm{dBm}$, the OSNR after the first span was $20.19 \mathrm{~dB}$ whereas the OSNR after the tenth span was $-1.39 \mathrm{~dB}$.
The OSNR at the first span was greater than the OSNR at the fifth span which was greater than the SNR at the tenth span no matter the value of input power as illustrated in fig. 2.

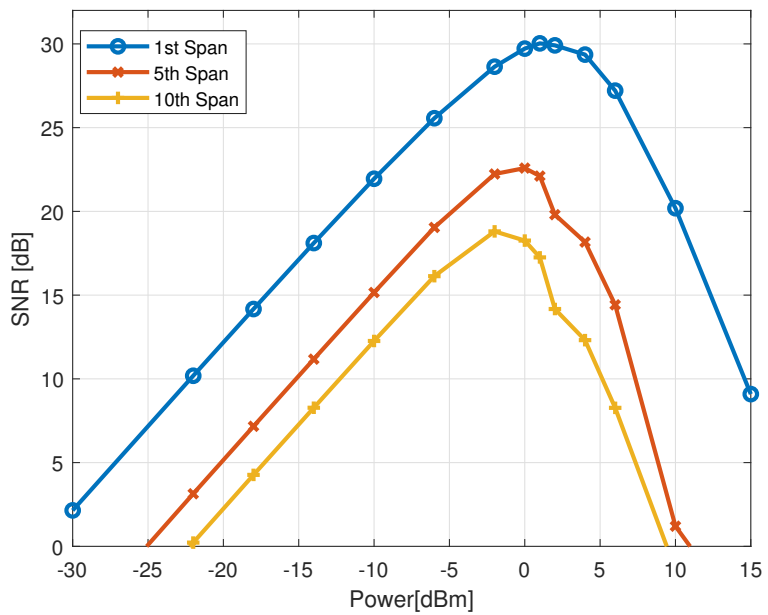

Fig. 1. OSNR vs Power comparison of a single channel system with at the 1st, 5th, and 10th Span. The RDPS value of the optical link is $0 \mathrm{ps} / \mathrm{nm}$.

For a WDM systems with 5 channels, OSNR reduced with an increase in the number of spans. The OSNR per channel increased with an increase in the input power until a certain threshold power was reached. Past the threshold power, the OSNR of the system reduced with an increase in power as shown in table 1 .

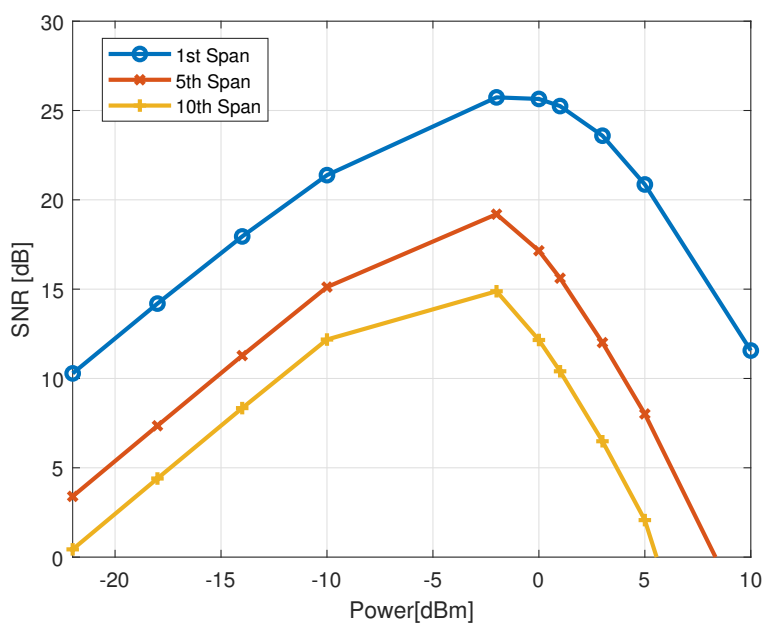

Fig. 2. OSNR vs Power comparison of a 5 channels WDM system at the 1st, 5th, and 10th Span. The RDPS value of the optical link is $0 \mathrm{ps} / \mathrm{nm}$.

An improved OSNR perfomance was observed when an optical link of $30 \mathrm{ps} / \mathrm{nm}$ RDSP was used. The perfomance was slightly equivalent to the 0 RDPS in the linear part but 
TABLE I

SNR VALUES (DB)AT DIFFERENT SPANS OF 0 RDPS WDM SYSTEM

\begin{tabular}{|c|c|c|c|}
\hline \multirow{2}{*}{$\begin{array}{c}\text { Power } \\
(\mathbf{d B m})\end{array}$} & \multicolumn{3}{|c|}{ OSNR in dB per Span } \\
\cline { 2 - 4 } & Span I & Span 5 & Span 10 \\
\hline-10 & 21.38 & 15.12 & 12.18 \\
\hline-2 & 25.74 & 19.20 & 14.88 \\
\hline 0 & 25.65 & 17.15 & 12.40 \\
\hline 1 & 25.25 & 15.62 & 10.40 \\
\hline 3 & 23.59 & 12.00 & 6.49 \\
\hline
\end{tabular}

TABLE II

OSNR VALUES (DB)AT DIFFERENT SPANS OF 30 RDPS WDM SYSTEM

\begin{tabular}{|c|c|c|c|}
\hline \multirow{2}{*}{$\begin{array}{c}\text { Power } \\
(\mathbf{d B m})\end{array}$} & \multicolumn{3}{|c|}{ OSNR in dB per Span } \\
\cline { 2 - 4 } & Span I & Span 5 & Span 10 \\
\hline-10 & 21.58 & 14.91 & 11.67 \\
\hline-2 & 26.28 & 18.91 & 14.56 \\
\hline 0 & 26.20 & 17.30 & 12.43 \\
\hline 1 & 25.77 & 15.96 & 10.88 \\
\hline 3 & 23.97 & 12.61 & 7.16 \\
\hline
\end{tabular}

an improvement was observed in the non-linear part as shown in fig. 4.

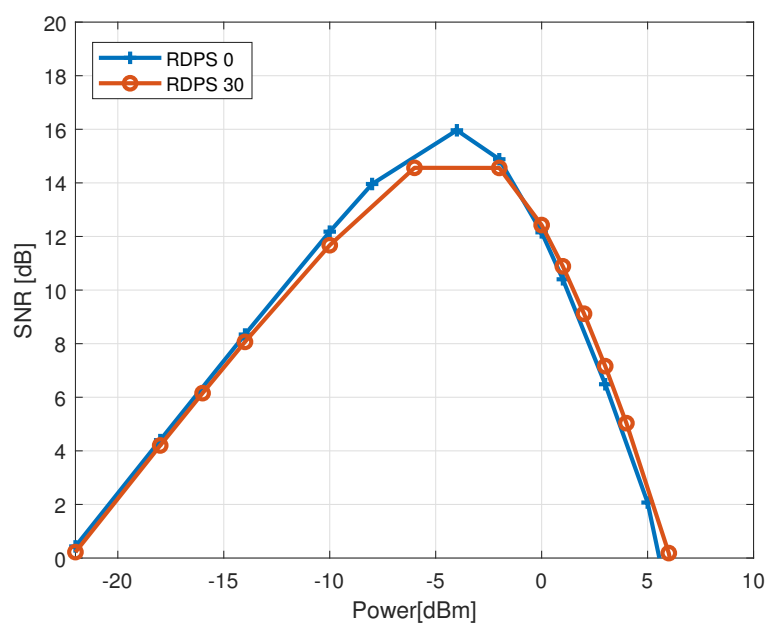

Fig. 3. OSNR vs Power comparison for a WDM system with varied RDPS value of the optical link - 5 Spans.

\section{RESUlts Discussion}

From the results presented in section III, we can draw conclusions that nonlinearity increases with distances and power. Higher OSNR values are recorded on the first few length of the optical link and the OSNR degrades as the distance grows. Linearity effects i.e. amplified spontaneous emission (ASE) dominates the link on the first few kilometers before it is outstripped by nonlinearity effects that are dependent on power and distance.

Self-phase modulation increases with an increase in a the number of spans which explains the degradation of OSNR for a phase modulated signal as shown below in the equation below.
The phase rotation after the 1st span is:

$$
A(l)=A(0) e^{-j \gamma L e f f(l)}|A(0)|^{2}
$$

The phase rotation after the 2 nd span is:

$$
A(2 l)=A(l) e^{-j \gamma \operatorname{Leff}(l)}|A(l)|^{2}
$$

which is equivalent to $A(0) e^{-j 2 \gamma L e f f(l)}|A(0)|^{2}$ and thus, after $\mathrm{N}$ span, the phase rotation is

$$
A(N l)=A(0) e^{-j N \gamma \operatorname{Leff}(l)}|A(0)|^{2}
$$

where

$$
\text { Leff }=\frac{1-e^{-\alpha z}}{\alpha}
$$

For cross phase mixing(XPM), a nonlinear phase shift in an optical field is experienced resulting from another field having a different wavelength, direction or state of polarization. This is experienced in a WDM systems with two or more channels. The phase rotation due to two power signals interacting with each other is

$$
\phi_{x p m}=-2 \gamma\left|A_{1}(0, \tau)\right|^{2} * h_{12}
$$

with the other signal being

$$
\phi_{\text {xpm }}=-2 \gamma\left|A_{2}(0, \tau)\right|^{2} * h_{12}
$$

where

$$
h_{12}(z, \tau)=\frac{1}{d_{12}} e^{\frac{-\alpha \tau}{d_{12}}} \Pi\left\{\frac{\tau+\frac{d_{12} Z}{2}}{Z\left|d_{12}\right|}\right\}
$$

and $\mathrm{d}_{12}=\beta_{1}-\beta_{2}$

$d_{12}$ is the walk-off parameter.

XPM just like SPM is additive, hence with an increase in number of spans $\mathrm{N}$, the total XPM experienced for channel 1 is:

$\phi_{X P M}(Z, \tau)=-2 \gamma P_{1}(0, t) \circledast \Sigma_{N} G_{0 N} h_{12}\left(\tau+\Sigma_{k=1}^{N-1} l_{k} d_{12}\right)$

The same is experienced with channel 2 .

$\phi_{X P M}(Z, \tau)=-2 \gamma P_{2}(0, t) \circledast \Sigma_{N} G_{0 N} h_{12}\left(\tau+\Sigma_{k=1}^{N-1} l_{k} d_{21}\right)$

with $G_{0 N}$ being the gain of the Nth amplifier.

DM fiber systems partially realign XPM contributionsopposite walk-off. This results in a lower impulse response and the overall XPM is the summation of all contributions. Compared to dispersion uncompensated systems, DM sytems have a smaller duration than DU and this explains the significance of DU systems where a strong value of dispersion is needed or left per span thus an improved OSNR as shown with 30 RDPS. Strong dispersion leads to strong low-pass filtering and therefore small XPM.

FWM depicted by equation 13 , results in power at any point along $\mathrm{z}$ being upper bounded by the product of mixing powers and the FWM kernel as shown in equation 16. 


\section{REFERENCES}

$A_{n}(Z)=e^{-\frac{\alpha}{2} Z}\left\{A_{n}(0)-j \gamma \Sigma_{k l m} A_{k}(0) A_{l}(0) A_{m}^{*}(0) \eta_{k l m}\right\}$

where

$$
\left|\eta_{k l m}\right|^{2}=\frac{1}{\alpha^{2}+\Delta \beta_{k l m n}^{2}}
$$

Power at any point is given by:

$$
P_{n}(Z)=\left|A_{n}\right|^{2}
$$

and is upper bounded by

$$
P_{n}(Z) \leq \gamma^{2} \Sigma_{k l m} P_{k}(0) P_{l}(0) P_{m}(0)\left|\eta_{k l m}\right|^{2}
$$

Big $\left|\Delta B_{k l m n}\right|$ can be used to mitigate the effects of FWM.

Gaussian noise model denoted by equation 17 , predicts the same performance with NLI tripling with the input power. The ASE noise which is independent on power is dominant in the linear part and thus explains high OSNR before it is dominated by NLI.

$$
O S N R=\frac{P}{N_{o} B+Q_{N L} P^{3}}
$$

The limitation of Gaussian Model is that it is modelled to predict performance in dispersion uncompensated fibers with unreliability in dispersion managed (DM) fibers; when the residual dispersion per span (RDPS) values are smaller than 60 $\mathrm{ps} / \mathrm{nm}$, the Gaussian model is considered unreliable as shown in [9].

\section{CONCLUSION}

In this paper, we investigated the influence of nonlinear interference on transmission and performance on a dispersion managed fiber with $\mathrm{Nx} 100 \mathrm{~km}$ spans. Conclusions can be drawn that NLI influences the OSNR of an optical link negatively with an increase in distance-OSNR reduces with an increase in number of spans due to accumulated NLI.

OSNR degrades in nonlinear part after an optimal power $P_{N L T}$ is surpassed. This explains the importance of choosing an appropriate power based on a user's preferred OSNR value.

An improvement in OSNR in nonlinear region was observed when a link of $30 \mathrm{ps} / \mathrm{nm}$ RDPS was used compared to when a $0 \mathrm{ps} / \mathrm{nm}$ RDPS link was used. This explains that having a strong value of dispersion in in an optical link is necessary to equalize the nonlinear interference experienced. Dispersion uncompensated (DU) optical link is thus preferable to dispersion managed (DM) in a long-haul optical transmission.
[1] A. Bononi, P. Serena, N. Rossi, E. Grellier, and F. Vacondio, "Modeling nonlinearity in coherent transmissions with dominant intrachannelfourwave-mixing," Opt. Exp., vol. 20, no. 7, pp. 7777-7791, 2012.

[2] P. Poggiolini, "The GN model of non-linear propagation and its applications," J. Lightw. Technol., vol. 32, no. 4, pp. 694-718, 2014.

[3] G. P. Agrawal, Nonlinear Fiber Optics, 3rd ed. (Academic, 2001).

[4] A. Ellis, M. McCarthy, M. AL Khateeb, M. Sorokina, and N. Doran, "Perfomance limits in optical communications due to fiber nonlinearity," Advances in Optics and Photonics., vol. 9, no. 3, pp. 431-475, 2017.

[5] R. Dar et al., "Inter-channel nonlinear interference noise in WDM systems: modeling and approaches for its cancellation," Journal of Lightwave Technology, early Access (2015).

[6] L. Hyung. "Analysis and characterization of fiber nonlinearities with deterministic and stochastic signal sources." Diss. Virginia Tech, 2000

[7] K. O. Hill, et al, "CW three wave mixing in single-mode optical fibers," J. Appl. Phys. 49, 5098-5106 (1978).

[8] P. Serena, Optilux Opensource Software. Downloadable from www.optilux.sourceforge.io

[9] P. Jenneve, P. Ramantanis, F. Boitier, N. Dubreuil,and S. Bigo, "Experimental investigation of the validity domain of the Gaussian Noise Model over dispersion managed systems", OFC 2016. 